\title{
Two-Tiered Network Design Architecture for Collision Avoidance Protocol in Wireless Sensor Networks
}

\author{
Widyasmoro \\ Department of Electrical Engineering, Universitas Muhammadiyah Yogyakarta \\ J1. Lingkar Selatan, Tamantirto, Kasihan, Yogyakarta, Indonesia \\ *Corresponding author, e-mail: widyasmoro@gmail.com
}

\begin{abstract}
Collision avoidance multiple access plays a significant role in Wireless Sensor Networks (WSN) as there may have a huge number of nodes in a network. Therefore, it has big chance to conflict when they want to send a packet to the server at the same time. In our work, we focus on the sensor networks with two-tier network design architecture that composed of simple function nodes and presented performance analysis of the proposed collision avoidance multiple access protocol for Wireless Sensor Networks, with emphasis on the contention process exercising in each contention subframe. Then we also examine the efficiency of the overall system based on the simulation results. The results shows that using different parameter of $n, m$, and $k$ then the successful probability tends to increase, however after certain value of $n$, the successful probability will decrease. Regarding the overall system efficiency, Esys, in order to get the optimum value of system efficiency, we have to maximize $\alpha$. It is obvious that the bigger value of $\beta$ will produce bigger value of $\alpha$. That is mean a shorter contention sub-frame leads to a better efficiency of the systems, if Lr (average length of transmission sub-frame) is fixed.
\end{abstract}

Keywords: Collision Avoidance, Multiple Access, Two-Tier Network Design, WSN, Contention Process, Efficiency

\section{Introduction}

Smart environments characterize by evolutionary advance in building, industrial, home, transportation systems automation, etc. The smart environment needs information about its environments as well as about its internal conditions. The information needed by smart environments is provided by Distributed Wireless Sensor Networks, which are responsible for sensing as well as for the first phases of the processing stage.

A wireless sensor network (WSN) is a network formed by a large number of sensor nodes where each node is equipped with a sensor to detect physical phenomena such as light, heat, pressure, etc. WSNs are regarded as a revolutionary information gathering method to build the information and communication system which will greatly improve the reliability and efficiency of infrastructure systems [1].

In a sensor networks, each node is a small sensor with a low capacity of processing, storage and energy. These networks are able to interact with their environment by sensing or controlling physical parameters; these nodes have to collaborate to fulfill their tasks which a single node is incapable of doing so; and they use wireless communication to enable this collaboration. In essence, the nodes without such a network contain at least some computation, wireless communication, and sensing or control functionalities.

A significant challenge in statistically multiplexed wireless networks is the collision problem, resulting from several nodes accessing the transmission channel simultaneously [2]. For example, the condition happened in an event-driven wireless sensor network, there are often hundreds to thousands of nodes deployed in a given area. When an event happens, many nodes will observe this event and send it to the server (sink). Hence, automatically many communications occur at the same time which implies an increase in the number of collisions [3]. Medium access control (MAC) 
protocols have been developed to take care of channel access. This problem is also known as channel allocation or multiple access problem.

The existing MAC protocols could be divided into two basic categories, scheduled protocols and contention based protocols. Scheduled based protocols for example used along with TDMA, FDMA, and CDMA that currently accepted as the cellular networks standard [4]. In other hand, another class of MAC protocols is based on contention which traditionally used by ALOHA [5] and carrier sense multiple access (CSMA) protocols [6].

Sensor networks can be differing from traditional wireless data networks in number of ways. Firstly, most nodes in sensor networks are battery powered, so it must be operating in as minimum as it could be. Secondly, nodes are often distributed in an adhoc fashion, so the topology of the networks itself are likely to be randomly distributed rather than organized network. Third, many applications employ large number of nodes and node density will vary in different places and times. Due to the characteristics explained before, many sensor oriented MAC protocols have been proposed, such as S-MAC [7], T-MAC [8], WiseMAC [9], D-MAC [10] and all of them are likely more focused on adhoc mesh sensor networks.

In our work, we propose a collision avoidance multiple access protocol for wireless sensor networks. The network itself will be considered has two-tier architecture. In our work, we focus on the performance measure of the proposed collision avoidance MAC protocol, with the emphasize on the contention process exercising in each contention sub-frame. We will derive the probability distribution of the number of successful mini-slot in the contention sub-frame. According to the distribution, we will examine performance measures such as channel efficiency by numerical results.

The rest of this work is organized as follows, Section 2 presents related work on wireless sensor networks, and then Section 3 presents the collision avoidance multiple access protocol. Section 4 describes the results and performance analysis, and the last Section 5 conclusion.

\section{Related Works}

Currently, wireless sensor networks are beginning to be deployed at an accelerated step. It is not unreasonable to expect that in 10-15 years that the world will be covered with wireless sensor networks with access to them via the Internet. This can be considered as the Internet becoming a physical network. This new technology is exciting with unlimited potential for numerous application areas including environmental, medical, military, transportation, entertainment, crisis management, homeland defense, and smart spaces. Since a wireless sensor network is a distributed real-time system a natural question is how many solutions from distributed and real- time systems can be used in these new systems? Unfortunately, very little prior work can be applied and new solutions are necessary in all areas of the system. The main reason is that the set of assumptions underlying previous work has changed dramatically. Most past distributed systems research has assumed that the systems are wired, have unlimited power, are not real-time, have user interfaces such as screens and mice, have a fixed set of resources, treat each node in the system as very important and are location independent. In contrast, for wireless sensor networks, the systems are wireless, have limited power, are real-time, utilize sensors and actuators as interfaces, have dynamically changing sets of resources, aggregate behavior is important and location is critical. Many wireless sensor networks also utilize minimal capacity devices which places a further strain on the ability to use past solutions.

For the WSNs, it important to consider the balance of requirements will be different from traditional (wireless) networks. Additional requirements come up, first and primary, the need to conserve energy. The importance of energy efficiency for the design of MAC protocols is relatively new and many of the "classical" protocols like ALOHA and CSMA contain no provisions toward this goal. Some researchers also consider covering energy aspects in MAC protocols. Other typical performance figures like fairness, throughput, or delay tend to play a minor role in sensor networks. Fairness is not important since the nodes in a WSN do not represent individuals competing for bandwidth, but they collaborate to achieve a common goal. The access or transmission delay performance is traded against energy conservation, and throughput is mostly not an issue either. Further important requirements for MAC protocols are scalability and robustness against frequent topology changes, as caused for example by nodes powering down temporarily to replenish their batteries by energy scavenging, mobility, deployment of new nodes, or death of existing nodes. The need for scalability is evident when considering very dense sensor networks with dozens or hundreds of nodes in mutual range. 
Based on various characteristics, MAC protocol is classified into two different types: ContentionBased and Contention-Free. Contention-free MAC is based on reservation and scheduling. Each node announces a time slot that it wants to use to the coordinator of the network. This coordinator schedules the request and allocates other nodes to their respective time slots. In this way, a node can access the channel without colliding with others because it is the only node which can transmit during its time slot. Bluetooth [11], TRAMA [12] and LEACH [13] are examples of this type of MAC. The technique guarantees low energy consumption because each node in the network works only in its time slot without collisions. However, the major disadvantage of this technique is that it is not well adaptable to topology change and is therefore non-scalable [14]. Any insertion or restraint of a node implies a time slot reallocation for all the nodes in the cluster.

In Contention-based protocols, a given transmit opportunity toward a receiver node can be taken by any of its neighbors. If only one neighbor tries its luck, the packet goes through the channel. If two or more neighbors try their luck, these have to compete with each other and in unlucky cases, for example, due to hidden-terminal situations, a collision might occur, wasting energy for both transmitter and receiver. There are two important contention-based protocols: (slotted) ALOHA and CSMA, along with mechanisms to solve the hiddenterminal problem.

\section{Collision Avoidance Multiple Access Protocol}

In the wireless communication networks, one of significant challenge is collision problem. Wireless Sensor Networks, in the deployment, will also form a such wireless communication networks, either in center controlled manner or in ad-hoc (decentralized) manner. The collision problem happens when several of nodes in the networks accessing the transmission channel simultaneously. Medium Access Control (MAC) protocols have been developed to handle the channel access problem, also known as channel allocation or multiple access problem. This layer (MAC) in the wireless networks protocol stack normally considered as a sub-layer of the data link layer.

The existing MAC protocols can be divided into two basic categories, there are scheduled protocols and contention based protocols. Scheduled based protocols for example are Time Division Multiple
Access (TDMA), Frequency Division Multiple Access (FDMA), and Code Division Multiple Access (CDMA). Those three protocols are normally used in current cellular networks and also known as collision-free protocol. However, those protocols are not commonly suggested for applied in wireless sensor network because of some reasons, such as hardware limitations and its limited computing power.

LEACH (Low Energy Adaptive Clustering Hierarchy) is an example of utilizing TDMA in wireless sensor networks. LEACH organizes nodes into cluster hierarchies, and applies TDMA within each cluster. The other group of MAC protocols is contention based protocols. A common channel is shared by all nodes and it is allocated on-demand. Terminals (nodes) have to compete among them for getting access to available channel, therefore they can transmit their packet. This contention based protocols, different with scheduled based protocols, is not pre-allocate the channel for a given terminal/ nodes to get access. Common examples of contention based MAC protocols are including ALOHA and Carrier Sense Multiple Access (CSMA) protocols. In the simple scheme (known as "pure ALOHA"), they permit terminals to transmit any time they desire. If, within some appropriate time-out period, they receive an acknowledgment from the destination, then they know that no conflicts occurred. Otherwise, they assume a collision occurred and they must retransmit. In CSMA scheme, terminals/nodes attempt to avoid collisions by listening to the carrier/ channel due to another terminal's transmission before they will transmit their packets. If a busy channel is detected, nodes will delay access and retry later.

In our work, we focus on sensor networks composed of transceiver nodes and the respective central controllers, as shown in Fig.1 below. This kind of networks will transmit and relayed sensing data from sensor nodes and then being collected by central controller. It is assumed that all the sensor nodes transmit small data units frequently. The node density also varies as a function of time due to node mobility. In such framework, collision problem should be specially considered for data collection of the central controller. We only consider the communication between central controller and onehop nodes. The data relaying between sensor nodes is out of our scope here.

In here, we analyze the performance of a collision avoidance multiple access protocol for sensor networks. The network considered has a two tie architecture, as shown in Fig.1. The red big 
circle in the picture depict as a Center Controller that will collect the sensed data from the nodes in the target area. The small blue and green circle around the center controller is representing nodes which are deployed and distributed in ad-hoc manner. In addition, the nodes with blue color (number 1 to 5) are nodes with one-hop range from center controller, positioned as intermediate node (IN) and the nodes with green color (number 6 to 10) act as common node in two-hop range from center controller. In the first tie, nodes operate in multi-hop manner by which any node has to forward sensed data to a nearest intermediate node (IN). A number of INs and a center controller forms the second tie connection. In the second tie, the center controller serves as the controller and forwards the sensed the sensed data from the INs to a networks server. The radio coverage range is considerably larger than one-hop range of a common node. With this two tie topology, each common node can be very simple and consumes very little power, although the INs and center controller may consume more power due to large radio coverage range is large. However, the number of INs and center controller is small, compared to the number of common nodes in the targeted area, therefore the two-tie architecture may provide synchronous or in line with the pre- requirement design of efficient MAC protocol for wireless sensor networks.

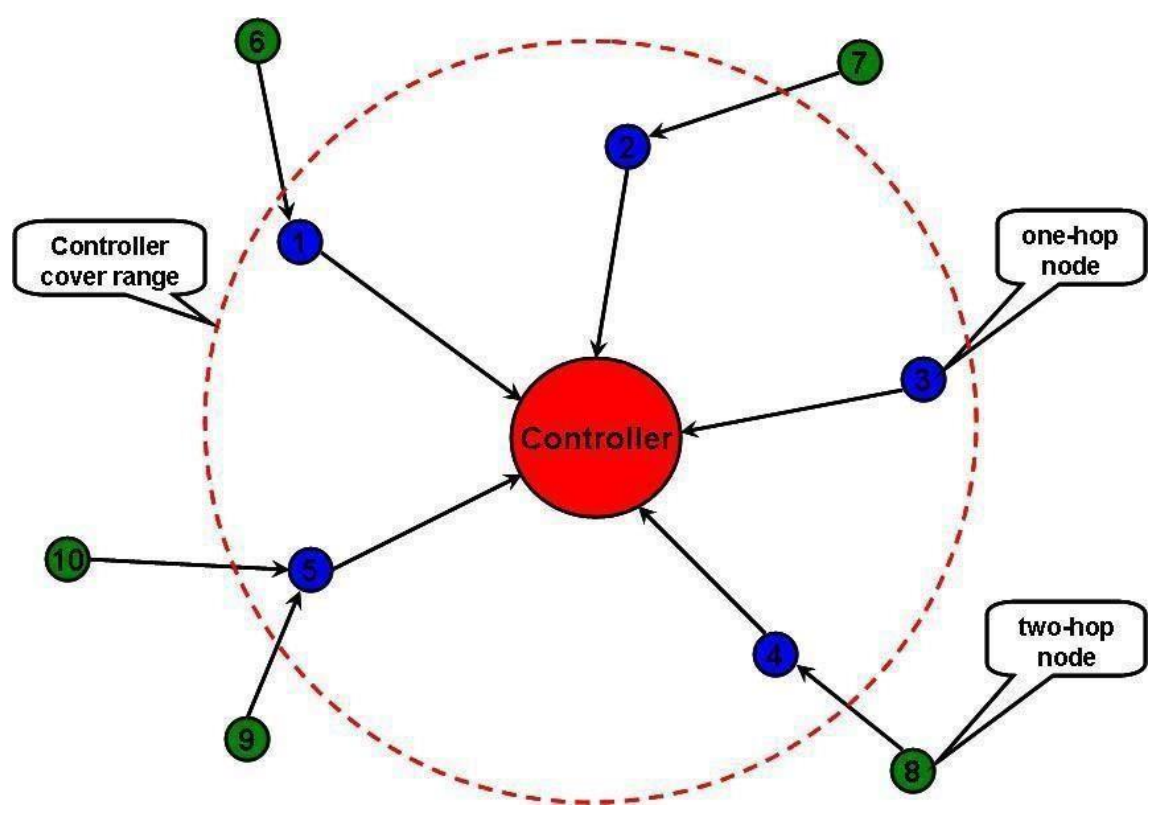

Fig. 1. Two-tie topology of the wireless sensor networks

For the first tie ad-hoc network, many sensororiented MAC protocols have been proposed, for example S-MAC, T-MAC, and D-MAC. In the rest of our discussion in this matter, we will focus on the second tie star network in which the center controller communicates with the INs within its radio coverage range by a shared radio channel. In such a framework, collision problem should be take a great part to discuss as considered for data collection of the center controller.

Further, the channel is operated in TDMA and TDD manner and the channel time is divided into frames. Each frame is further divided into contention sub-frame and data transmission subframe. The first slot in the frame is the frame start slot by which the controller declares the starting of a frame and broadcasts the number of mini-slots,
Nc, that follow used for the INs to transmit reservation request. Each IN acquires the Nc value and randomly chooses one from among the $\mathrm{Nc}$ mini-slots to transmit the request packet. Following the contention mini-slots is a contention result broadcasting slot which is used by center controller to broadcast the contention result to all the INs. After the contention process finishes, the resultant mini-slots fall into three categories: blank slot, which means no INs selected the slots; successful slot, means exactly one IN selected the slot; collision slot, which means more than one INs selected the slot. According to the contention result broadcast from the controller, all the INs selected a successful slot are allowed to send data packets in the data transmission sub-frame, in the order they sent the request packet. Obviously, in each slot of 
the transmission sub-frame only one IN is allowed to transmit packet; thus, the data packet collision is avoided.

In the contention sub-frame, before the process of "successful slot" transmit their packet, all active INs will get in to the contention process and thus will determine the resultant of each slot by three categories: blank slot (no INs selected the slots), successful slot (exactly one IN selected the slot), collision slot (more than one INs selected the slot). Further, in the contention process we will treat as the occupancy problem, that is, randomly distributing $\mathrm{k}$ distinct balls into $\mathrm{n}$ distinct boxes. This method seemingly ordinary problem that has a vast number of applications and can be apply in our work, that is, multiple access problem.

We consider mini-slots as boxes and INs as balls. The contention process corresponds to randomly distribute balls into boxes and the successful minislots corresponds to the boxes each contain exactly one ball. For this treatment, we define $\mathrm{P}(\mathrm{x} ; \mathrm{k}, \mathrm{n})$ as the probability that $\mathrm{x}$ boxes each contain exactly one ball resulting from randomly distributing $\mathrm{k}$ ball into $\mathrm{n}$ boxes.

We define $P(m ; k, n)$ as the probability that $m$ boxes each have exactly one ball resulting from distributing $k$ balls into $n$ boxes. Our aim is to specify the probability distribution of $P(m ; k, n)$. Obviously, placing $k$ balls into $n$ boxes will result in $n^{k}$ different ways, and each way can be classified into one of the events of the probability space. So, we define $S(m ; k, n)$ as

$$
P(m ; k, n)=\left(1 n^{k}\right) S(m ; k, n)
$$

where the definition for the arguments of $S(m$; $k, n)$ are the same as those for $P(m ; k, n)$. If the function $S(m ; k, n)$ can be determined, so the required probability is also can be specified.

Then, after we get the numerical value of $P(m$; $k, n)$, then we can find out the expected number (mean value) of "successful slot/correct slot" as follows.

$$
\eta=\sum i \times P(i ; k, n)
$$

with $\mathrm{k}$ and $\mathrm{n}$ as the similar meaning as we define $\mathrm{P}(\mathrm{m} ; \mathrm{k}, \mathrm{n})$ abovementioned.

As we know, after the contention process finished, all the INs selected a successful slot are allowed to send data packets in the data transmission sub-frame, in the order they sent the request packet. In this data transmission phase, it is obviously collision free phase, because in each slot of the transmission sub-frame only one IN is allowed to transmit packet. Then, we will determine the system efficiency, Esys, as follows,

$$
E_{s y s}=\frac{(\eta \times L r)}{(n \times L c)+(\eta \times L r)}
$$

where $\mathrm{Lr}$ is the average length of transmission subframe; $\mathrm{n}$ is the number of minislots in the contention subframe; Lc is the length of contention subframe; and $\eta$ is the expected number (mean value) of "successful slot/correct slot".

\section{Performance Analysis and Numerical Results}

In our work, we examine the performance of the proposed collision avoidance MAC protocol, by mathematical analysis and computer simulation. In this section, we will focus on the results and explanation of our proposed protocol. First we will discuss about contention process exercising, particularly happen in the contention sub-frame. And the second one, we will also give some explanations and result related with the system efficiency Esys. We simulates work with different number of nodes / terminals (k) as well as some different number of mini-slots (n) for the proposed. We use some of the parameters in the simulation process as shown in Table 1.

TABLE I

SIMULATION PARAMETERS

\begin{tabular}{lc}
\hline \hline \multicolumn{1}{c}{ Simulation Parameter } & Value \\
\hline $\begin{array}{l}\text { Size of system, } \mathrm{N} \\
\text { Number of contention } \\
\text { minislots in the frame }\end{array}$ & between 2 to 30 \\
$\begin{array}{l}\text { Number of successful } \\
\text { minislots in the frame }\end{array}$ & 1 to 13 \\
$\beta=L_{r} / L_{c}$ & $2 ; 5 ; 10$ \\
\hline \hline
\end{tabular}

As above mentioned, we define $P(m ; k, n)$ as the probability that $m$ successful slot resulting from the certain number of $k$ terminals which contending to get access to the $n$ minislots in the current frame. Our aim is to specify the probability distribution of $P(m ; k, n)$. Table 2 shows that using different parameter of $n, m$, and $k$ then the successful probability tends to increase, however after certain value of $n$, the successful probability will decrease.

Figure 2 also confirms that we could get optimum value for number of mini-slots in the 
system, it is adjacent to the highest value of successful probability in the y-axis, for $m=2$ and $k=10$ the optimum value of $n$ is 6 , and for $m=5$ and $k=20$ is 13 .

As we discuss in the Section 3 about system efficiency, then it is interesting also to know overall system efficiency for the proposed scheme. In equation 3 we already mentioned our definition of system efficiency. And then, the equation 3 can be represent in another way below:

$$
E_{s y s}=\frac{\left(\frac{L r}{L c \times n}\right) \times \eta}{1+\eta \times\left(\frac{L r}{L c \times n}\right)}
$$

Let we define $\alpha=\left(\frac{L r}{L c \times n}\right) \times \eta$ and $\beta=\left(\frac{L r}{L c}\right)$ . In order to maximize $E_{s y s}$, we have to maximize $\alpha$. It is obvious that the bigger value of $\beta$ will produce bigger value of $\alpha$. In other word, a shorter contention subframe leads to a better efficiency of the systems, if $L r$ is fixed. We can observe from Table 3 below that when we increase the value of $\beta$ to the system, then the system efficiency, $E_{s y s}$, also will increase.

Table 3 depicts to us that the successful probability $P$ with some variations of value of $k$ and then we also calculate the expected number for each $k$. Also we can see from table 4 below that when we increase the value of $\beta$ to the system, then the system efficiency, Esys, also will increase.

TABLE II

NUMERICAL RESULTS FOR $P(M ; K, N)$ WITH DIFFERENT $M$ AND $K$

\begin{tabular}{cccc}
\hline \hline $\begin{array}{c}\text { Number of } \\
\text { Minislot) }\end{array}$ & $\mathrm{m}=2 ; \mathrm{k}=10$ & $\mathrm{~m}=5 ; \mathrm{k}=20$ & $\mathrm{~m}=10 ; \mathrm{k}=30$ \\
\hline 1 & - & - & - \\
2 & 0 & - & - \\
3 & 0.0046 & - & - \\
4 & 0.1236 & - & - \\
5 & 0.3370 & 0 & - \\
6 & 0.3508 & $3.0532 \mathrm{e}-009$ & - \\
7 & 0.2970 & $1.6030 \mathrm{e}-005$ & - \\
8 & 0.2351 & 0.0012 & - \\
9 & 0.1822 & 0.0154 & - \\
10 & 0.1408 & 0.0639 & 0 \\
11 & 0.1094 & 0.1332 & $6.8730 \mathrm{e}-017$ \\
12 & 0.0856 & 0.1902 & $3.1785 \mathrm{e}-011$ \\
13 & 0.0677 & 0.2203 & $4.1124 \mathrm{e}-008$ \\
\hline \hline
\end{tabular}

TABLE III

SUCCESSFUL PROBABILITY AND EXPECTED NUMBER FOR EACH K

\begin{tabular}{ccccc}
\hline \hline $\begin{array}{c}\boldsymbol{m} \\
\begin{array}{c}\text { Number of successful } \\
\text { mini-slot) }\end{array}\end{array}$ & $\mathrm{n}=5 ; \mathrm{k}=10$ & $\mathrm{n}=5 ; \mathrm{k}=20$ & $\mathrm{n}=5 ; \mathrm{k}=30$ & $\mathrm{n}=5 ; \mathrm{k}=50$ \\
\hline 0 & 0.1707 & 0.7271 & 0.9538 & 0.9991 \\
1 & 0.4056 & 0.2576 & 0.0460 & $8.9199 \mathrm{e}-004$ \\
2 & 0.3370 & 0.0152 & $2.1360 \mathrm{e}-004$ & $2.2003 \mathrm{e}-008$ \\
3 & 0.0840 & $9.3984 \mathrm{e}-005$ & $3.5106 \mathrm{e}-008$ & $1.8634 \mathrm{e}-015$ \\
4 & 0.0026 & $6.0964 \mathrm{e}-009$ & $3.5311 \mathrm{e}-015$ & $3.1115 \mathrm{e}-028$ \\
5 & 0 & 0 & 0 & 0 \\
$\boldsymbol{\eta}$ (Expected Number) & 1.3420 & 0.2883 & 0.0464 & $8.9203 \mathrm{e}-004$ \\
\hline \hline
\end{tabular}


TABLE IV

EFFICIENCY SYSTEM WITH DIFFERENT VALUE OF $B$

\begin{tabular}{ccccc}
\hline \hline $\boldsymbol{\beta}$ & $\begin{array}{c}\mathrm{k}=10 \mathrm{n}=5 ; \\
\eta=1.3420\end{array}$ & $\begin{array}{c}\mathrm{k}=20 \mathrm{n}=5 ; \\
\eta=0.2883\end{array}$ & $\begin{array}{c}\mathrm{k}=30 \mathrm{n}=5 ; \\
\eta=0.0464\end{array}$ & $\begin{array}{c}\mathrm{k}=50 \mathrm{n}=5 ; \\
\eta=8.9203 \mathrm{e}-004\end{array}$ \\
\hline 10 & 0.7286 & 0.3657 & 0.0849 & 0.0018 \\
15 & 0.8010 & 0.4638 & 0.1222 & 0.0027 \\
20 & 0.8430 & 0.5356 & 0.1565 & 0.0036 \\
25 & 0.8703 & 0.5904 & 0.1883 & 0.0044 \\
\hline \hline
\end{tabular}

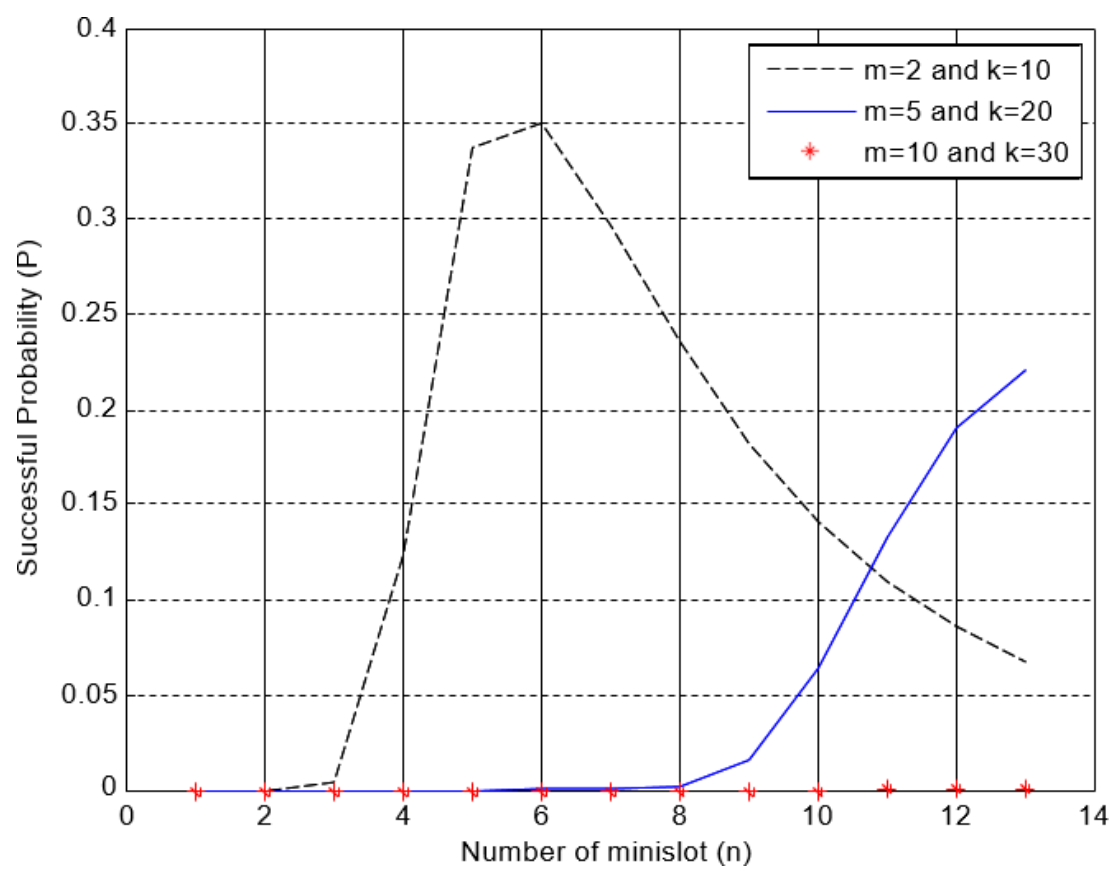

Fig. 2. Successful probability adjacent to number of minislots

\section{Conclusion}

In our work, we proposed two-tier network design architecture for collision avoidance multiple access protocols in Wireless Sensor Networks, with emphasis on the contention process exercising in each contention subframe. We consider the network has two tier architecture, which the first tier operate in multi-hop manner and the second tier, consist of intermediate node (IN) and center controller operated in a TDMA and TDD manner. In our proposed scheme, we treat the contention process as an occupancy problem.

The results shows that using different parameter of $\mathrm{n}, \mathrm{m}$, and $\mathrm{k}$ then the successful probability tends to increase, however after certain value of $n$, the successful probability will decrease. Regarding the system efficiency, Esys, in order to get the optimum value of system efficiency, we have to maximize $\alpha$. It is obvious that the bigger value of $\beta$ will produce bigger value of $\alpha$. In other word, a shorter contention subframe leads to a better efficiency of the systems, if $\operatorname{Lr}$ (average length of transmission subframe) is fixed.

\section{References}

[1] Shu Yinbiao, et al, "Internet of Things: wireless Sensor Networks," International Electrotechnical Commision White Paper, July 20014.

[2] Wei Ye and John Heidemann, "Medium Access Control in Wireless Sensor Networks," USC / ISI Technical Report ISI-TR-580, October 2003.

[3] H-C. Lee, H. Guyennet, N. Zerhouni, "A New Contention Access Method for Collision Avoidance in Wireless Sensor Networks" IEEE, 2007.

[4] T.S. Rappaport, Wireless Communications, Principles and Practice, Prentice Hall, 1996.

[5] Norman Abramson, "Development of The ALOHANET," IEEE Transactions on Information Theory, Vol. IT-31, No. 2, March 1985.

[6] Leonard Kleinrock and Fouad Tobagi, "Packet Switching in Radio Channels: Part I- Carrier Sense 
Multiple Access Modes and Their Throughput Delay

Characteristics," IEEE Transactions on

Communications, Vol. 23, No. 12, pp. 1400-1416,

Dec. 1975.

[7] Wei Ye, Heidemann J, Estrin D; "An EenergyEfficient MAC Protocol for Wireless Sensor Networks," In INFOCOM, New York, June. 2002.

[8] T. V. Dam and K. Langendoen; "An Adaptive Energy-Efficient MAC protocol for Wireless Sensor Networks," 1st ACM Conf. Embedded Networked Sensor Sys., Los Angeles, CA, Nov. 2003.

[9] C. C. Enz, A. El-Hoiydi, J-D. Decotignie, V. Peiris, "WiseNET: An Ultralow- Power Wireless Sensor Network Solution," IEEE Computer, Volume: 37, Issue: 8, August 2004.

[10] G. Lu, B. Krishnamachari, and C. S. Raghavendra; "An Adaptive Energy-Efficient and Low-Latency MAC for Data Gathering inWireless Sensor Networks," Proc.18th International Parallel and Distribution Processing Symposium, Apr. 2004, p. 224.

[11] Specification of the Bluetooth System: Core (2001). [Online]. Available http://www.bluetooth.org/.

[12] Koen Langendoen, Preprint of A Book Chapter in Medium Access Control in Wireless Sensor Networks, Volume II: Practice and Standards, Nova Science Publishers, 2007.

[13] Fan Yiming and Yu Jianjun, "Communication Protocol for Wireless Sensor Network About LEACH," 2007 International Conference on Computational Intelligence and Security Workshops, 2007.

[14] Wen, Jyh-Horng \& Wang, Jyu-Wei. (1999). An occupancy problem involved in multiaccess communication systems. Applied Mathematics Letters. 12. 57-60.

\section{Authors' information}

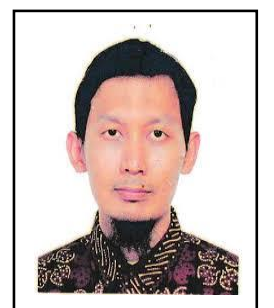

Widyasmoro was born on May 11, 1983 in Cilacap, Central Java, Indonesia. He is currently serves as Lecturer in the Department of Electrical Engineering, Faculty of Engineering, Universitas Muhammadiyah Yogyakarta, Indonesia. $\mathrm{He}$ received his Bachelor Degree from the Departement of Electrical Engineering, Universitas Jenderal Soedirman, Indonesia in 2007 and he got his Master Degree from the Departement of Computer Science and Information Engineering, Asia University, Taiwan, in 2009. His research interests are in the area of wireless communications, including 4G and 5G technology, Internet of Things, and the implementations of artificial intelligence. 